\title{
Misguided multiplication: Creating false memories with numbers rather than words
}

\author{
BRYAN J. PESTA \\ Cleveland State University, Cleveland, Ohio \\ and \\ RAYMOND E. SANDERS and MARTIN D. MURPHY \\ University of Akron, Akron, Ohio
}

\begin{abstract}
We built Deese (1959)/Roediger and McDermott (1995) (DRM) false memory lists composed of multiplication problems rather than words. Half these lists contained table-related, near neighbors (e.g., $3 \times 7=$ ??, $3 \times 9=$ ??) of a missing multiplication answer lure (e.g., 24). The other half contained problems unrelated to the lure (e.g., $5 \times 5=$ ??, $11 \times 3=$ ??). Participants solved each problem in a single list and then took immediate recognition (Experiment 1) or recall and then recognition tests (Experiment 2) for the answers. Many people misremembered that the lure was an answer to a study-phase problem, but only when solving the study list that contained the lure's neighbors. False memory was also greater for some list-lure combinations than others, as seen previously with words. We have thus demonstrated that numbers can also produce false memory, and we use the mental math and DRM task literatures to explain these results.
\end{abstract}

Research on false memory has increased dramatically, since Roediger and McDermott's (1995) revival of a task invented by Deese (1959). In the task, participants hear a study list whose items are strong associates of a nonpresented theme word, called the critical lure. With sleep as the critical lure, for example, the study list would contain these items: bed, rest, awake, tired, dream, wake, snooze, blanket, doze, slumber, snore, and nap. After hearing the list, participants complete a recall or a recognition test for the study items. This simple task manipulation (i.e., leave the critical lure out of the study list) produces impressive false memory. Typically, people "remember" the lure about as often as they do target items, at least with the best lists available (see Stadler, Roediger, \& McDermott, 1999). These memory errors occur with high confidence, and with many people specifically remembering (Tulving, 1985 ) words that never appeared in the study phase of the experiment (see Roediger, McDermott, \& Robinson, 1998, for a review).

Research on the Deese (1959)/Roediger and McDermott (1995) (DRM) task has focused on identifying boundary conditions for when false memory does and does not occur. For example, not all DRM lists produce strong effects (Stadler et al., 1999), and manipulations like presenting the study items visually instead of verbally (Smith \& Hunt, 1998) weaken the effect. However, if false memory occurs when people systematically report remembering items that were not present at study, then research

Correspondence should be addressed to B.J. Pesta, 14724 Grapeland, Cleveland, OH 44111 (e-mail: bpesta22@cs.com). shows false memories for (1) pictures as well as words (Koutstaal \& Schacter, 1997); (2) phonemic as well as semantic list-lure relationships (Sommers \& Lewis, 1999); and (3) emotional (e.g., rape) as well as nonemotional words, even when the study lists contain no emotional words (Pesta, Murphy, \& Sanders, 2001).

Our present goal was to see if numeric stimulimultiplication problems and their answers - can also produce false memory. As with standard DRM lists, where the study items are strongly related to a single word, it is possible to compile multiplication lists where the study problems are strongly related to a single multiplication answer. In these lists, the study items will all be table related, near neighbors of a lure. Neighbors are problems that equal the lure after adding (or subtracting) 1 to either of the problem's operands (see Ashcraft, 1992). For example, $3 \times$ $7=21$ neighbors the answer 24 , because adding 1 to its second operand results in a new multiplication problem whose answer is 24 (i.e., $3 \times 8=24$ ). Indeed, the multiplication answer 24 has 10 problems that are neighbors. We wondered if people would misremember this answer as a critical lure after having solved its 10 neighbor problems at study.

Why might solving table-related multiplication problems lead a person to misremember a multiplication answer, and what might this effect tell us about false memory in the DRM task? An interesting parallel exists between the justification for using multiplication problems as stimuli here, and at least one explanation for word-based false memory in the DRM task. In the mental arithmetic literature, a well documented finding is that solving a neighbor problem can prime reaction time (RT) to verify a target an- 
swer (Campbell \& Tarling, 1996). The explanation for this effect appeals to activation spreading in semantic memory networks. For example, in Ashcraft's (1992) network retrieval model, multiplication problems and their answers are stored in semantic memory as nodes in an associative network. Associative strength determines the semantic distance between nodes, with neighbor problems stored closest together. When a person solves a multiplication problem, activation from the operands spreads to the target answer node, and then to its neighbors. So, solving the problem $3 \times 7=$ ?? would first activate the target node 21 , and then, to a smaller extent, the neighbor nodes 24,18 , 14 , and 28.

This spreading activation process can also influence performance on episodic memory tests. Pesta, Sanders, and Murphy (1999) accurately predicted the episodic recall of target multiplication answers on the basis of the number of neighbor problems solved in the same stimulus set. In four experiments, people remembered target answers better when the study list contained many of their neighbors than when it did not. In the present study, though, the activated neighbor would be a critical lure, and we explored whether the process of neighbor activation outlined in the network model is strong enough to produce false recall or false recognition of that lure.

Spreading activation may also explain word-based false memory. But, instead of activation spreading to table-related multiplication answers, it spreads to the semantic associates of the study list items. So, study words in a DRM list would automatically activate the critical lure, making it more familiar and perhaps even inducing it to enter working memory (see Schacter, Verfaellie, \& Pradere, 1996). At test, false memory may occur if the participant attributes the lure's high degree of familiarity to having experienced it in the study list.

Spreading activation models seem to offer a reasonable account of false memory in the DRM task with words, and predict the presence of the effect with numbers (i.e., multiplication answers). Therefore, our present goal was to demonstrate that solving multiplication problems can indeed produce false memory of a multiplication answer lure. In Experiment 1, we developed study lists containing problems that either were or were not table-related neighbors of a single multiplication answer lure (i.e., 24). Subjects solved the problems in one of these lists at study and then took an immediate recognition test for the answers and the critical lure. In Experiment 2, we expanded on this design to test the effect's generality by developing 10 new study lists and by exploring false memory of multiplication answer lures in both recall and in recognition.

\section{EXPERIMENT 1}

\section{Method}

Participants and Design. The participants were 40 undergraduates (6 males and 34 females) with a mean age of 28.0 (range = 18-46) years. The design was a $2 \times 3$ factorial with study list group (lure-activated list, lure-not-activated list) between subjects and item type (target, critical lure, unrelated lure) within subjects. We ran the experiment in small groups, randomly assigning each group to one of the two study list conditions.

Materials. We created two 10-item study lists. In one, each tobe-solved multiplication problem neighbored the answer 24 , our critical lure (i.e., $3 \times 7=$ ??, $3 \times 9=$ ??, $6 \times 3=$ ??, $6 \times 5=$ ??, $8 \times 2=$ ??, $8 \times 4=? ?, 12 \times 1=? ?, 12 \times 3=? ?, 4 \times 5=? ?$, and $4 \times 7=$ ??). For these study items, the answer 24 results by adding or subtracting 1 to one of each problem's operands. In the second study list, the to-be-solved problems did not activate the answer 24 as a near neighbor (i.e., $2 \times 7=$ ??, $11 \times 3=$ ??, $10 \times 2=$ ??, $3 \times 5=$ ??, $4 \times$ $4=? ?, 3 \times 10=? ?, 5 \times 5=? ?, 2 \times 5=? ?, 5 \times 7=? ?$, and $8 \times 5=$ ??). We presented the study items without their answers, forcing the participants to solve each problem, because we were looking for the strongest effects possible. In Pesta et al. (1999), neighbor activation moderated target-answer memory best when people solved versus read each problem at study. Finally, because product size also moderates memory for multiplication answers (see Pesta et al., 1999), we constrained the range of products to be similar across lists (12-36 in the activated list and 10-40 in the nonactivated list).

The one-page recognition test contained seven randomly ordered items: three targets (selected randomly from each list), one critical lure (24), and three unrelated distractors. The unrelated distractors were multiplication answers (in the same product size range as the study items) that were not activated multiple times as neighbor answers of the study list problems. Using this criterion, however, we could find only two distractors that were unrelated to the study items in both lists (i.e., 9 and 22). The third distractor was different for the two lists (i.e., 10 in the activated list, 36 in the nonactivated list).

The recognition test items appeared in a single column. Next to each item was a blank space that participants used for their old (i.e., "I solved this answer") or new (i.e, "I did not solve this answer") ratings. For any answer rated "old," participants checked a box corresponding either to a remember (i.e., "I have a vivid, specific memory of solving for this answer") or to a know (i.e, "I'm sure I solved for this answer, but I have no specific memory of doing so") response.

Procedure. We told participants that they (1) would hear a series of multiplication problems, (2) should solve each problem "in their heads" as they heard it, and (3) should try to memorize each answer as they solved it. We began the experiment by reading the multiplication problems aloud, pausing $3 \mathrm{sec}$ between each. After reading the last problem, we distributed the recognition tests face down, and we explained the difference between old versus new and remember versus know responses (adopting our instructions after those outlined in Roediger \& McDermott, 1995). The recognition test was not timed, and when everyone finished, we reread the study list and had participants generate the answer to each problem on paper. These data allowed us to rule out solution errors as contributors to the recognition results. No one incorrectly solved a study problem by answering with the critical lure (e.g., $3 \times 7=24$ ).

\section{Results and Discussion}

We used $p=.05$ as the alpha level for all analyses. Table 1 shows the recognition results by study list group and item type. A $2 \times 3$ mixed analysis of variance (ANOVA) on the proportion-old data revealed significant effects of study list group $\left[F(1,38)=9.33, M S_{\mathrm{e}}=\right.$ $.09]$, item type $\left[F(2,76)=53.70, M S_{\mathrm{e}}=.09\right]$, and the group $\times$ item type interaction $\left[F(2,76)=15.39, M S_{\mathrm{e}}=\right.$ .09]. Because each participant studied only one list, ratings for the lure data are binary (however, target and unrelated distractor data are not binary). We therefore also conducted a chi square on the lure data. In the activated group, 15 of 20 people misremembered the lure, whereas 
Table 1

Recognition Results in Experiments 1 and 2 by List Type and Item Type

\begin{tabular}{llll}
\hline & \multicolumn{3}{c}{ Proportion of Old Responses } \\
\cline { 2 - 4 } Item Type & Overall & Remembered & Known \\
\hline Experiment 1 & & & \\
Lure activated & & .76 & .04 \\
Target & $.80^{\mathrm{a}}$ & .55 & .20 \\
Lure & $.75^{\mathrm{a}}$ & .03 & .10 \\
Unrelated & $.13^{\mathrm{b}}$ & & \\
Lure not activated & & .79 & .09 \\
$\quad$ Target & $.88^{\mathrm{a}}$ & .05 & .10 \\
Lure & $.15^{\mathrm{b}}$ & .02 & .13 \\
Unrelated & $.15^{\mathrm{b}}$ & & \\
Experiment 2 & & & \\
Lure activated & & .64 & .10 \\
Target & $.74^{\mathrm{b}}$ & .49 & .19 \\
Lure & $.49^{\mathrm{c}}$ & .30 & .06 \\
Unrelated & $.14^{\mathrm{d}}$ & .08 & .10 \\
Lure not activated & & & .08 \\
Target & $.82^{\mathrm{a}}$ & .72 & .10 \\
Lure & $.17^{\mathrm{d}}$ & .09 & .07 \\
Unrelated & $.17^{\mathrm{d}}$ &
\end{tabular}

Note-Within experiments, means not sharing superscripts are significantly different via Tukey LSD.

only 3 of 20 did so in the not-activated group $\left[\chi^{2}(1)=\right.$ 12.2]. Hence, people were more likely to call the lure "old" when the study list contained its neighbors than when it did not.

In contrast, Tukey LSD tests show that study list group did not significantly affect proportion-old ratings either for targets $[t(38)=1.25]$ or for unrelated distractors $[t(38)=1.0]$. Also, proportion-old values did not differ reliably between targets and lures in the lure-activated group $(t<1.0)$. This last finding parallels the standard false memory effect seen with words.

The ANOVA results for the proportion-remembered data mirror those above: significant effects of study list group $\left[F(1,38)=13.91, M S_{\mathrm{e}}=.06\right]$, item type $[F(2,76)=$ $\left.62.19, M S_{\mathrm{e}}=.09\right]$, and the group $\times$ item interaction $\left[F(2,76)=9.37, M S_{\mathrm{e}}=.09\right]$. Post hoc tests revealed that people in the activated group remembered the lure about as often as they did targets- the difference in proportionremembered ratings to lures versus targets was nonsignificant $[t(19)=1.33]$. Note, however, that our power was less than ideal in this comparison since it used only half our sample (i.e., the 20 participants in the lure-activated group only). In contrast, people in the nonactivated group simply did not remember the lure (i.e., proportion remembered $=.05$ ), with a significant difference between targets and lures $[t(19)=11.72]$.

Experiment 1 produced robust false recognition of a multiplication answer lure, but only when people solved its neighbors. This effect was not due to wild guessing or to some unknown property of the lure that made it prone to false alarms. Using false alarms to unrelated distractors as a baseline (i.e., $13 \%$ and $15 \%$ in the activated and nonactivated lists, respectively), false memory of the lure when its neighbors were not studied (i.e., 15\%) was at the floor. We have thus extended the DRM illusion of memory into the realm of numbers, since $55 \%$ of people in the activated group (compared with 5\% in the nonactivated group) reported a "vivid, specific memory" of having generated the critical lure as an answer to a study problem, when in fact they had not.

\section{EXPERIMENT 2}

In Experiment 1, we demonstrated false memory of only a single multiplication answer, leaving the effect's generality in question. In Experiment 2 we therefore added 10 new multiplication DRM lists to our design (for 12 lists total). We built these 12 lists around six multiplication answer lures; half our lists activated a single lure, and the other half served as control conditions for that lure. We thus hoped to replicate the Experiment 1 results with a broader set of stimuli. Also, in Experiment 1, we demonstrated the effect using only recognition. In Experiment 2, we had participants solve the problems in a study list and then take an immediate recall test, followed by a recognition test, for the answers and the critical lure.

\section{Method}

Participants and Design. The participants were 158 undergraduates (43 males, 115 females) with a mean age of 26.1 (range = $18-40$ ) years, run in groups of $12-15$ people per study list. We presented only one math list to each group because presenting multiple lists would have resulted in instances where the study problems in one list could have "cross-activated" the lure in another list. Thus, the design was a $2 \times 3$ factorial, with study list group (lure activated or not activated) between subjects and item type (target, critical lure, unrelated) within subjects. Note that this design again produces recognition data for the lures (but not for the targets or unrelated distractors) that are binary.

Materials. We searched for multiplication answers in the $2 \times 2$ through $12 \times 12$ table that had many near neighbors, finding these six answers: $12,18,24,30,36$, and 40 . For each of these lures, we compiled two study lists. One list contained only its neighbors, and the other list contained problems in roughly the same product size range as its matched list, but with none of these problems neighboring the lure. The Appendix presents all 12 study lists. Note that these lists contain 8 to 10 items each, whereas, word-based DRM lists typically contain 15 items. This difference would be expected to weaken false memory here relative to word-based studies.

For the recognition test, we selected unrelated distractors separately for each list using the same criteria employed in Experiment 1 . In many cases our unrelated distractors had fewer neighbors than did our lures, and this difference may have reduced the baseline false alarm rate to these items. The key comparison, however, for demonstrating false memory of a multiplication answer lure is the difference in false alarm rates when the study list does and does not contain its neighbors. Finally, the recognition test was identical in layout to that used previously, except that here we had 12 different tests corresponding to the 12 different study lists.

Procedure. As in Experiment 1, we read the instructions to participants and then read each multiplication problem, pausing $3 \mathrm{sec}$ between each. Thereafter, we distributed a blank page and had participants recall the answers to the study list problems in any order. After $2 \mathrm{~min}$, we collected the recall sheets and distributed the recognition tests. Since no one incorrectly solved a study problem with the lure in Experiment 1, we opted not to have participants re- 
Table 2

Mean Proportion (and Standard Deviations) Target and Lure Recall, and the Mean Number of Unrelated Intrusions in Experiment 2 by List Type and Item Type

\begin{tabular}{lccccc}
\hline & \multicolumn{2}{c}{ Lure Activated } & & \multicolumn{2}{c}{ Lure Not Activated } \\
\cline { 2 - 3 } \cline { 5 - 6 } Item Type & $M$ & $S D$ & & $M$ & $S D$ \\
\hline Targets & $.69^{\mathrm{a}}$ & .17 & $.72^{\mathrm{a}}$ & .16 \\
Lures & $.26^{\mathrm{b}}$ & .44 & $.03^{\mathrm{c}}$ & .16 \\
Unrelated $^{*}$ & 0.41 & .63 & 0.49 & .72 \\
\hline
\end{tabular}

Note-Means not sharing superscripts differ significantly via Tukey LSD. *These data constitute raw numbers as opposed to proportions (e.g., in the lure-not-activated lists, roughly one intrusion other than the lure occurred for every 2 participants).

solve the study problems here. All other aspects of the procedure were identical to those of Experiment 1.

\section{Results}

Recall. Table 2 shows mean proportion target and lure recall, and the mean number of unrelated intrusions for the lure-activated and not-activated conditions (the Appendix provides these data on a list by list basis). A 2 (activated, not activated) $\times 2$ (target, lure) ANOVA revealed significant effects of study group $\left[F(1,156)=11.5, M S_{\mathrm{e}}=\right.$ $.06]$, item type $\left[F(1,156)=335, M S_{\mathrm{e}}=.07\right]$, and the group $\times$ item interaction $\left[F(1,156)=18.7, M S_{\mathrm{e}}=.07\right]$. Target answer recall did not differ across the activation groups $(t<1.0)$. Lure intrusions, however, occurred $26 \%$ of the time in the activated group and only $3 \%$ of the time in the not-activated group. This difference was significant $\left[\chi^{2}(1)=14.22\right]$. Finally, recall intrusions for all nonlure answers combined were .41 (i.e., almost one non-lure intrusion for every 2 participants). However, the highest intrusion rate for any single non-lure answer was only .08 . That is, for any given list, a specific non-lure answer was rarely intruded on by more than 1 of our participants.

Recognition. Table 1 shows the recognition results by study group and item type for Experiment 2. A $2 \times 3$ ANOVA revealed effects of study group $[F(1,156)=4.92$, $\left.M S_{\mathrm{e}}=.11\right]$, item type $\left[F(2,312)=161, M S_{\mathrm{e}}=.10\right]$, and the group $\times$ item interaction $\left[F(2,312)=18.4, M S_{\mathrm{e}}=.10\right]$. A Tukey LSD showed somewhat poorer target recognition in the activated versus nonactivated group $[t(156)=$ 2.12]. We have no explanation for this effect, but note that it did not also emerge in the remember/know analyses below, and that it does not alter our conclusions about false memory of multiplication answer lures. Post hoc tests also revealed no difference in false alarms to the unrelated distractors across the activated and nonactivated groups $[t(156)=1.01]$, but higher lure false recognition in the activated group $\left[\chi^{2}(1)=17.8\right]$. Consistent with this last finding, only in the activated group were false alarms to the lures higher than false alarms to the unrelated distractors $[t(81)=5.80]$.

The $2 \times 3$ ANOVA on the proportion-remembered data yielded conclusions identical to those for the proportionold data, with one exception. Namely, the proportion remembered values for targets did not differ across the lure activated and not-activated groups $[t(156)=1.56, p=.12]$.
In sum, multiplication answer lures produced fairly strong false recognition, since almost half our participants misremembered solving the lure when the study list contained its neighbors, and most of these false alarms corresponded to remember, rather than to know, responses. In contrast, only $17 \%$ of the participants had lure false alarms when the study list did not activate the lure.

Experiment 2 appeared to produce weaker false recognition than did Experiment 1. False alarm rates were .75 and .49 , respectively. Further, in Experiment 1, 73\% (i.e., $55 \% / 75 \%$ ) of people who rated the lure "old" in the activated group also rated it as specifically remembered, compared with only $33 \%$ (i.e., $5 \% / 15 \%$ ) of people in the nonactivated group. In Experiment 2, however, this difference was smaller: $61 \%$ (i.e., $30 \% / 49 \%$ ) in the activated group, and 53\% (9\%/17\%) in the nonactivated group. In an attempt to explain this across-experiment difference, we looked at false recognition rates separately for each of our six lures (presented in the Appendix). Interestingly, false recognition of the multiplication lures seemed to vary by list, as previously found with words (Stadler et al., 1999). The lure answer 24 produced the strongest effects (47\% recall intrusions and $60 \%$ false alarms in the activated group); and the lure answer 40 produced the weakest effects (15\% recall instructions and $36 \%$ false alarms in the activated group). False recognition rates for the common lure, 24 , were similar in the two experiments $(75 \%$ vs. $60 \%$ ). Thus, the weaker false recognition in Experiment 2 seems largely due to the new lists.

Finally, as Roediger and McDermott (1995) found with words, false recognition of a multiplication answer lure was indeed more likely when the participant first falsely recalled it. In the activated groups, almost everyone (i.e., 91\%) who misrecalled a lure also false alarmed to it; but only $33 \%$ of people who did not misrecall a lure nonetheless false alarmed to it.

\section{GENERAL DISCUSSION}

From the participant's standpoint, the present experiments were trivially simple. Each person solved 8 to 10 multiplication problems and then took an immediate recall and/or recognition test for the answers. Nonetheless, many people in the activated group reported specific but false recollections of generating the multiplication lure as an answer to a study phase problem. Hence, we have a clear demonstration that numbers, like words, can produce false memory in the DRM task. To our knowledge, this is also the first demonstration of false memory where a person misremembers performing a dynamic mental process (i.e., solving for an answer) as opposed to the more static memory error of misremembering a presented word.

An unresolved issue in the DRM task concerns the necessary and sufficient conditions needed for the production of false memory. This issue revolves around Underwood's (1965) implicit associative response explanation for false memory, which today exists in two versions (Roediger \& McDermott, 1995). In the explicit/conscious version, the conscious generation of the critical lure at 
study produces false memory at test (see Schacter et al., 1996, for a discussion; see also Johnson, Hashtroudi, \& Lindsay, 1993). In this view, false memories may arise from failures in reality monitoring, where people mistake prior thoughts of the critical lure for prior perception of its occurrence. In the implicit/nonconscious version, though, false memory can occur even when the lure does not come to the participant's mind at study. Here, feelings of familiarity-perhaps triggered by activation spreading through a semantic network-are sufficient to produce the effect (see Roediger \& McDermott, 1995, and Schacter et al., 1996, for discussions). False memories arise when people misattribute the lure's familiarity to having experienced it in the study list.

The present evidence with word list remember/know judgments tends to support the explicit model, because participants most often report remembering the lure's presentation. Remember responses imply that people explicitly generated the lures as they experienced the study lists (see Schacter et al., 1996, pp. 329-330). With math lists, however, the relationship between the study items and the critical lure is far less obvious than with semantic word lists (e.g., asking someone to free-associate to table would likely produce many responses, including the critical lure chair; asking someone to free-associate to $3 \times$ $7=$ ?? would likely produce little more than the answer $21)$. It seems doubtful that many people explicitly generated our math lures as they solved the problems in their study list. Hence, the false memory effects we observed may have been driven primarily by implicit processes (i.e., familiarity, caused by residual spreading activation from a target answer to its neighboring lure). The use of more subtle list-lure themes may allow future research to separate the explicit and implicit components behind false memory.

Our justification for using multiplication answers as stimuli appealed to the mental math RT literature. Given that neighbor problems can prime RT to verify target answers, we thought it also possible that neighbor problems could prime a lure to the point that people misremembered solving for it in a study list. Perhaps any primetarget relationship producing priming in the RT literature can also produce false memory in the DRM task. For example, Roediger and McDermott's "semantic" false memory parallels the semantic priming effect (Meyer \& Schvaneveldt, 1971), where processing a prime word (e.g., nurse) facilitates processing of semantically related target words (e.g., doctor). In addition, phonemic (Sommers \& Lewis, 1999) and orthographic (Schacter, Verfaellie, \& Anes, 1997) list-lure relationships also have counterparts in the RT literature (Forster \& Taft, 1994).

Schacter et al. (1996) have argued that the mental processes capable of producing accurate memory and comprehension also seem capable of producing false memory. Activating related concepts based on current experience is usually adaptive, and people often use various scripts or schemas to make sense of the world (Schank \& Abel- son, 1977). But, as the DRM task shows, these same processes can lead to memory error. The information automatically activated by experiencing an event may be falsely linked to the episodic memory of that event. Here, solving the study list problems likely led to the automatic activation of the neighboring lures, which in turn produced false memory of these lures. Perhaps the price we pay for accurately comprehending and integrating complex information over time is a difficulty in determining where, when, and how we experienced any single piece of the information.

\section{REFERENCES}

Ashcraft, M. H. (1992). Cognitive arithmetic: A review of data and theory. Cognition, 44, 75-106.

CAMPBEll, J. I. D., \& TARLING, D. P. M. (1996). Retrieval processes in arithmetic production and verification. Memory \& Cognition, 24, 156-172.

DEESE, J. (1959). On the prediction of occurrence of particular verbal intrusions in immediate recall. Journal of Experimental Psychology, 58, 17-22.

Forster, K. I., \& TAFT, M. (1994). Bodies, antibodies, and neighborhooddensity effects in masked form priming. Journal of Experimental Psychology: Learning, Memory, \& Cognition, 20, 844-863.

Johnson, M. K., Hashtroudi, S., \& Lindsay, D. S. (1993). Source monitoring. Psychological Bulletin, 114, 3-28.

KoutstaAl, W., \& Schacter, D. L. (1997). Gist based false recognition of pictures in older and younger adults. Journal of Memory \& Language, 37, 555-583.

Meyer, D. E., \& Schvaneveldt, R. W. (1971). Facilitation in recognizing pairs of words: Evidence of a dependence between retrieval operations. Journal of Experimental Psychology, 90, 227-234.

Pesta, B. J., Murphy, M. D., \& Sanders, R. E. (2001). Are emotionally charged lures immune to false memory? Journal of Experimental Psychology: Learning, Memory, \& Cognition, 27, 328-338.

Pesta, B. J., Sanders, R. E., \& Murphy, M. D. (1999). A beautiful day in the neighborhood: What factors determine the generation effect for simple multiplication problems? Memory \& Cognition, 27, 106115 .

Roediger, H. L., III, \& McDermott, K. B. (1995). Creating false memories: Remembering words not presented in lists. Journal of Experimental Psychology: Learning, Memory, \& Cognition, 21, 803-814.

Roediger, H. L., III, McDermott, K. B., \& Robinson, K. J. (1998) The role of associative processes in creating false memory. In M. A. Conway, S. E. Gathercole, \& C. Cornoldi(Eds.), Theories of memory II (pp. 187-245). Hove, U.K.: Psychological Press.

Schacter, D. L., Verfaellie, M., \& Anes, M. D. (1997). Illusory memories in amnesic patients: Conceptual and perceptual false recognition. Neuropsychology, 11, 331-342.

Schacter, D. L., Verfaellie, M., \& Pradere, D. (1996). The neuropsychology of memory illusions: False recall and recognition in amnesic patients. Journal of Memory \& Language, 35, 319-334.

Schank, R. C., \& Abelson, R. P. (1977). Scripts, plans, goals and understanding. Hillsdale, NJ: Erlbaum.

Smith, R. E., \& Hunt, R. R. (1998). Presentation modality affects false memory. Psychonomic Bulletin \& Review, 5, 710-715.

Sommers, M. S., \& LewIS, B. P. (1999). Who really lives next door: Creating false memories with phonological neighbors. Journal of Memory \& Language, 40, 83-108.

Stadler, M. A., Roediger, H. L., III, \& McDermott, K. B. (1999). Norms for word lists that create false memories. Memory \& Cognition, 27, 494-500.

Tulving, E. (1985). Memory and consciousness. Canadian Psychologist, 26, 1-12.

UNDERWOOD, B. J. (1965). False recognition produced by implicit verbal responses. Journal of Experimental Psychology, 70, 122-129. 


\section{APPENDIX}

Study Items Used in Experiment 2

(Within Lists, Each Item Was Presented in a Fixed Random Order)

12-activated: $1 \times 6,4 \times 4,3 \times 5,3 \times 3,4 \times 2,6 \times 3,2 \times 12,1 \times 11,2 \times 7,2 \times 5$ 12-control: $\quad 1 \times 8,2 \times 2,2 \times 3,9 \times 2,3 \times 7,1 \times 9,2 \times 8,10 \times 1,5 \times 4,11 \times 2$

18-activated: $2 \times 8,2 \times 10,1 \times 9,3 \times 9,3 \times 5,3 \times 7,2 \times 6,4 \times 6$

18-control: $\quad 1 \times 8,2 \times 5,7 \times 4,3 \times 10,7 \times 2,11 \times 1,5 \times 5,11 \times 2$

24-activated: $3 \times 7,3 \times 9,6 \times 3,6 \times 5,8 \times 2,8 \times 4,12 \times 1,12 \times 3,4 \times 5,4 \times 7$

24-control: $\quad 2 \times 7,11 \times 3,10 \times 2,3 \times 5,4 \times 4,3 \times 10,5 \times 5,2 \times 5,5 \times 7,8 \times 5$

30-activated: $3 \times 9,3 \times 11,4 \times 6,6 \times 6,2 \times 10,4 \times 10,5 \times 5,5 \times 7$

30-control: $\quad 2 \times 9,4 \times 7,7 \times 6,3 \times 7,8 \times 4,2 \times 11,5 \times 9,4 \times 11$

36-activated: $6 \times 7,6 \times 5,9 \times 5,9 \times 3,2 \times 12,4 \times 12,3 \times 11,4 \times 10,4 \times 8$

36-control: $\quad 5 \times 7,4 \times 11,3 \times 10,6 \times 4,5 \times 8,4 \times 7,7 \times 3,7 \times 7,11 \times 2$

40-activated: $4 \times 8,6 \times 8,3 \times 10,5 \times 10,5 \times 7,5 \times 9,4 \times 11,4 \times 9$

40-control: $3 \times 9,5 \times 6,12 \times 4,4 \times 7,7 \times 6,11 \times 3,6 \times 9,7 \times 7$

Recall and Recognition Rates in Experiment 2

\begin{tabular}{lccccccc}
\hline & & \multicolumn{2}{c}{ Proportion Recalled } & & \multicolumn{2}{c}{ Proportion Recognized } \\
\cline { 6 - 7 } & $n$ & Targets & Lure & & Targets & Lure & Unrelated \\
\hline 12-activated & 15 & .69 & .20 & & .80 & .60 & .11 \\
12-control & 13 & .80 & .08 & & .90 & .23 & .26 \\
18-activated & 14 & .80 & .29 & .76 & .36 & .07 \\
18-control & 12 & .76 & .00 & .75 & .33 & .19 \\
24-activated & 15 & .69 & .47 & .64 & .60 & .02 \\
24-control & 12 & .73 & .08 & .92 & .25 & .14 \\
30-activated & 13 & .63 & .15 & .80 & .56 & .21 \\
30-control & 14 & .68 & .00 & .69 & .07 & .07 \\
36-activated & 12 & .66 & .25 & .75 & .42 & .28 \\
36-control & 13 & .75 & .00 & .92 & .00 & .15 \\
40-activated & 13 & .63 & .15 & .69 & .36 & .18 \\
40-control & 12 & .60 & .00 & .75 & .17 & .25 \\
\hline
\end{tabular}

(Manuscript received December 28, 1998;

revision accepted for publication January 26, 2001.) 